BULL. AUSTRAL. MATH. SOC.

MOS $12 \mathrm{KO5}$

VOL. $5(1971), 275-280$.

\title{
The sub-near-field structure of finite near-fields
}

\section{Susan Dancs}

The sub-near-field structure of finite near-fields is analogous to the sub-field structure of finite fields. A finite near-field

of order $p^{2 n}$ contains a unique sub-near-field of order $p^{\lambda}$ if and only if $\lambda$ divides $l n$.

\section{Introduction}

A (left distributive) near-field is a structure which satisfies all the axioms for a skew field except possibly right distributivity.

A pair of positive integers $q, n$ satisfying the relations

(1) $q=p^{2}$ for some prime $p$;

(2) each prime divisor of $n$ divides $q-1$;

(3) if $q \equiv 3 \bmod 4$, then $n \neq 0 \bmod 4$;

is called a Dickson number pair.

The results of Ellers and Karzel [2] and Zassenhaus [4] show that there is a uniform method for constructing a finite near-field of order $q^{n}$, with centre of order $q$, from the field of order $q^{n}$, whenever $q, n$ is a Dickson number pair. The near-field obtained by this construction is called a Dickson near-field; $q$ and $n$ will be called its invariants. Moreover, with seven exceptions, all finite near-fields are Dickson. A list of the exceptional cases can be found on p. 391 of $\mathrm{Hall}$ [3].

Received 10 May 1971. Communicated by M.F. Newman. The author thanks Dr M.F. Newman for his help and encouragement with this work. 
The results of this paper, together with those of [1], make it possible to describe completely the sub-near-field structure of finite near-fields.

For the exceptional cases this is easy. Each exceptional finite near-field has order $p^{2}$ and thus has precisely two sub-near-fields: the near-field itself and its prime field of order $p$ (which is not necessarily central).

Let $N$ be a sub-near-field of a finite Dickson near-field $K$, with invariants $q=p^{2}, n$. Since additively $N$ is a subgroup of $K$, the order $|N|$ of $N$ is $p^{\lambda}$, for some $\lambda$. Since multiplicatively $N^{*}$ is a subgroup of $K^{*}, p^{\lambda}-1$ divides $p^{2 n}-1$ and hence $\lambda$ divides $2 n$. It follows (Theorem of [1]) from the construction of $K$, that for every $\lambda$ dividing $I_{n}$, there is a sub-near-field of $K$ of order $p^{\wedge}$, which is a Dickson near-field, with invariants $p^{\prime}, \lambda / Z^{\prime}$, where $Z^{\prime}$ is the greatest common divisor of $Z I$ and $\lambda$, and $I$ is the solution of $I \equiv\left(p^{2 n}-1\right) /\left(p^{\lambda}-1\right) \bmod n$ such that $0<I \leq n$.

In this paper the following result is proved.

THEOREM. For each $\lambda$ dividing $2 n$, a Dickson near-field of order $p^{2 n}$ contains at most one sub-near-field of order $p^{\lambda}$.

Thus an analogous result to that for finite fields has been obtained. A finite near-field of order $p^{2 n}$ has a unique sub-near-field of order $p^{\lambda}$, if and only if $\lambda$ divides $2 n$. Further, the structure of each sub-near-field can be completely specified.

\section{Number-theoretic lemma}

The proof of the theorem depends on the following number-theoretic result.

LEMMA 2.1. Let $g, h$ be positive integers, $h \neq 1$. If every prime $r$ which divides $p^{g h}-1$ also divides $p^{g}-1$, then $p$ is a Mersenne prime, $g=1$ and $h=2$.

Proof. It can readily be seen that, for all $k \geq 2$, there exists 
$f \geq 1$, such that

$$
p^{g k}-1=\left(p^{g}-1\right)\left[f\left(p^{g}-1\right)+k\right]
$$

Let $s$ be a prime divisor of $h, w=\left(p^{g s}-1\right) /\left(p^{g}-1\right)$ and $r$ a prime divisor of $w$. Then $r \mid p^{g s}-1$ and hence $r \mid p^{g h}-1$. Thus, by assumption, $r \mid p^{g}-1$. But $w=f\left(p^{g}-1\right)+s$, for some $f$. Hence $r \mid s$ and, consequently, $r=s$. Thus $w=s^{u}$ with $u>1$, since both $f$ and $g$ are non-zero.

If $s$ is an odd prime, then

$$
\begin{aligned}
s^{u} & =w=1+p^{g}+\ldots+p^{g(s-1)} \\
& =\left(p^{g}-1\right)\left[s^{\prime}\left(p^{g}-1\right)+\sum_{i=1}^{s-1} i\right]+s \text {, by (*), } \\
& =\left(p^{g}-1\right)\left[s^{\prime}\left(p^{g}-1\right)+s(s-1) / 2\right]+s \\
& \equiv s \bmod s^{2}, \text { since } s \mid p^{g}-1,
\end{aligned}
$$

contradicting $u>1$. Hence $s=2$, and $w=p^{g}+1$. Since $u>1$, it follows that $p^{g} \equiv 3 \bmod 4$. Hence $p, g$ are odd. But then

$$
2^{u}=p^{g}+1=(p+1)\left(p^{g-1}-p^{g-2}+\ldots-p+1\right)
$$

implies $g=1$ and $p$ is a Mersenne prime. Since 2 is the only prime divisor of $h, h=2^{v}$. If $v>I$, then $r \mid p^{4}-1$ would imply $r \mid p^{h}-1$. But

$$
\begin{aligned}
\left(p^{4}-1\right) /(p-1) & =\left(p^{2}+1\right)(p+1)=\left(2^{2 u}-2^{u+1}+2\right) 2^{u} \\
& =2^{u+1}\left[2^{u}\left(2^{u-1}-1\right)+1\right]
\end{aligned}
$$

so $p^{4}-1$ would have an odd prime divisor dividing both $\left[2^{u}\left(2^{u-1}-1\right)+1\right]$ and $p-1=2\left(2^{u-1}-1\right)$, which is clearly impossible. Thus $v=1$, $h=2$.

\section{Proof of the theoremi}

LEMMA 3.1 (Ellers und Karzel [2], Satz 1). The multiplicative 
group, $K^{*}$, of a finite Dickson near-field of order $q^{n}$, has two generators $a$ and $b$ which satisfy the following relations:

(4) $a^{m}=1, b^{n}=a^{t}, b a b^{-1}=a^{q}$, where $m, n, t$ and $q$ satisfy the following conditions:

(5) $m=\left(q^{n}-1\right) / n$;

(6) $t=m /(q-1)$;

(7) $q^{n} \equiv 1 \bmod m$ and, if $n>1, q^{\nu} \neq 1 \bmod m$ for $1 \leq v<n$;

(8) $(n, t)=(q-1, t) \leq 2$;

(9) $(n, t)=(q-1, t)=2$ if and only if $n \equiv 2 \bmod 4$ and $q \equiv 3 \bmod 4$.

Furthermore, (see Hall [3], p. 390)

(10) a Syzow subgroup of $K^{*}$ of odd order is cyclic. A Sylow 2-subgroup of $K^{*}$ is cyclic or generalized quaternion.

For the remainder of this paper, $A$ will denote the normal subgroup of $K^{*}$, of order $\left(p^{2 n}-1\right) / n$, generated by $a$. Further, let 2 be the (unique) subgroup of $A$ of order $q-1$. Note $Z$ is the centre of $K^{*}$.

LEMMA 3.2. Let $r$ be a prime divisor of $q-1$ and $r^{s}$ the highest power of $r$ dividing $q-1$. For all positive integers $v \leq s$, the group $K^{*}$ has a unique subgroup of order $r^{v}$.

Proof. Since 2 is normal in $K^{*}$, the Sylow $r^{\text {-subgroup, }} R$, of 2 is contained in every Sylow $r$-subgroup, $S$, of $K^{*}$.

If $S$ is cyclic, the result follows. If $S$ is not cyclic, $r=2$ and $(n, t)=2$. Then, by (9), $s=1$ and $|R|=2$. But a generalized quaternion group has a unique subgroup of order 2 and, again, the result follows.

With these lemmas, the theorem can now be proved.

Let $N$ be a sub-near-field of $K$, of order $p^{\lambda}$, and $N^{*}$ the multiplicative group of $N$. 
Let $\bar{l}=(z, \lambda)$ and let $Z^{\prime}$ be the (unique) subgroup of $z$ of order $p^{\bar{l}}-1$. Since $\left(p^{2}-1, p^{\lambda}-1\right)=\left(p^{\bar{l}}-1\right), N^{*} \cap Z \leq Z^{\prime}$. Further, if $r^{v} \mid p^{\bar{\tau}}-1$, for some prime $r$, then $r^{v} \mid p^{\lambda}-1$ and, by Lemma $3.2, N^{*}$ contains a unique subgroup $R_{v}$, of order $r^{v}$. Hence $R_{v} \leq Z^{\prime}$ and so $\left|N^{*} \cap Z\right|=\left|Z^{\prime}\right|=p^{\bar{l}}-1$ and $N^{*} \cap Z=Z^{\prime}$.

Hence $p^{\lambda}-1=\left(p^{\bar{\tau}}-1\right) t^{\prime} n^{\prime \prime}$, where $\left|N^{*} \cap A\right|=\left(p^{\bar{\imath}}-1\right) t^{\prime}$ and $n^{\prime \prime} \mid n$.

Let $M$ also be a sub-near-field of $k$, of order $p^{\lambda}$. Without loss of generality, it may be assumed that $\left|M^{*} \cap A\right| \geq\left|N^{*} \cap A\right|$. Since $(n, t) \leq 2,\left|M^{*} \cap A\right|$ is $\left|N^{*} \cap A\right|$ or $2\left|N^{*} \cap A\right|$, and so $M^{*} \cap A \geq N^{*} \cap A$, since $A$ is cyclic. Therefore, $N^{*} \cap A \leq(N \cap M)^{*} \leq N^{*}$. Hence $|(N N M) *|=\left(p^{\lambda}-1\right) / \mu$, where $\mu \mid n^{n}$ and hence $\mu \mid n$.

But $N \cap M$ is a sub-near-field of $K$. Thus $\left(p^{\lambda}-1\right) / \mu=p^{\nu}-1$, for some $\nu$. Further, since $N^{*} \cap Z \leq(N \cap M)^{*} \leq N^{*}, \bar{z} \mid \nu$ and $\nu \mid \lambda$.

If $r$ is a prime divisor of $p^{\lambda}-1=\mu\left(p^{\nu}-1\right)$, then either $r \mid p^{\nu}-1$ or $r \mid \mu$. If $r \mid \mu$, then $r \mid n$ and, by (2), $r \mid p^{l}-1$. Hence $r \mid p^{\bar{l}}-1$. So $r \mid p^{\nu}-1$

Thus, by Lemma 2.1, either $\lambda=\nu$ and $N=M$, or $p$ is a Mersenne prime, $\lambda=2$ and $v=1$. If $p=2^{u}-1$, with $u>1$, then $p \equiv 3 \bmod 4$. Further, since $l=\bar{l}=(l, \lambda), l$ is odd and $q \equiv 3 \bmod 4$. Since $\lambda \mid I_{n}, n$ is even. Thus, by (3), $n \equiv 2 \bmod 4$. But $p^{\lambda}-1=p^{2}-1=(p-1)(p+1)=(p-1) 2^{u}$. Thus $\left|N^{*} \cap A\right| \geq(p-1) 2^{\mathcal{u}-1}$, contradicting $|(N \cap M) *|=p-1$, since $u>1$. Thus $N=M$ and the proof of the theorem is complete.

\section{References}

[1] Susan Dancs, "On finite Dickson near fields", Abh. Math. Sem. Univ. Homburg (submitted). 
[2] Erich Ellers und Helmut Karzel, "Endliche Inzidenzgruppen", Abh. Math. Sem. Univ. Hamburg 27 (1964), 250-264.

[3] Marshall Hall, Jr, The theory of groups (The Macmillan Company, New York, 1959).

[4] Hans Zassenhaus, "Über endliche Fastkörper", Abh. Math. Sem. Hansisch. Univ. 11 (1936), 187-220.

Institute of Advanced Studies, Australian National University, Canberra, ACT. 